\title{
COMPUTATIONAL PROOFS OF CONGRUENCES \\ FOR 2-COLORED FROBENIUS PARTITIONS
}

\author{
DENNIS EICHHORN and JAMES A. SELLERS
}

Received 13 April 2001

\begin{abstract}
In 1994, the following infinite family of congruences was conjectured for the partition function $c \phi_{2}(n)$ which counts the number of 2-colored Frobenius partitions of $n$ : for all $n \geq 0$ and $\alpha \geq 1, c \phi_{2}\left(5^{\alpha} n+\lambda_{\alpha}\right) \equiv 0\left(\bmod 5^{\alpha}\right)$, where $\lambda_{\alpha}$ is the least positive reciprocal of 12 modulo $5^{\alpha}$. In this paper, the first four cases of this family are proved.
\end{abstract}

2000 Mathematics Subject Classification: 05A17, 11P83.

1. Background and introduction. In his 1984 Memoir of the American Mathematical Society, Andrews [2] introduced two families of partition functions, $\phi_{k}(m)$ and $c \phi_{k}(m)$, which he called generalized Frobenius partition functions. In this paper, we will focus our attention on one of these functions, namely $c \phi_{2}(m)$, which denotes the number of generalized Frobenius partitions of $m$ with 2 colors. In [2], Andrews gives the generating function for $c \phi_{2}(m)$ :

$$
\sum_{m \geq 0} c \phi_{2}(m) q^{m}=\frac{\left(q^{2} ; q^{4}\right)_{\infty}}{\left(q ; q^{2}\right)_{\infty}^{4}\left(q^{4} ; q^{4}\right)_{\infty}},
$$

where $(a ; b)_{\infty}=(1-a)(1-a b)\left(1-a b^{2}\right)\left(1-a b^{3}\right) \cdots$. Andrews then proves the following: for all $n \geq 0$,

$$
\begin{aligned}
& c \phi_{2}(5 n+3) \equiv 0(\bmod 5), \\
& c \phi_{2}(2 n+1) \equiv 0(\bmod 4) .
\end{aligned}
$$

More recently, Sellers [9] conjectured the following infinite family of congruences satisfied by $c \phi_{2}$.

CONJECTURE 1.1. For all $n \geq 0$ and $\alpha \geq 1$,

$$
c \phi_{2}\left(5^{\alpha} n+\lambda_{\alpha}\right) \equiv 0\left(\bmod 5^{\alpha}\right)
$$

where $\lambda_{\alpha}$ is the least positive reciprocal of 12 modulo $5^{\alpha}$.

The case $\alpha=1$ is (1.2).

The reader will note the similarity of this conjecture to the well-known family of congruences for $p(m)$, the classical partition function of $m$ : for all $n \geq 0$,

$$
p\left(5^{\alpha} n+\gamma_{\alpha}\right) \equiv 0\left(\bmod 5^{\alpha}\right),
$$


where $\gamma_{\alpha}$ is the least positive reciprocal of 24 modulo $5^{\alpha}$. (For two different proofs of $(1.5)$, see $[1,6]$.) Unfortunately, (1.4) has proven to be much more difficult to prove than (1.5).

The goal of this paper is to prove the following theorem.

THEOREM 1.2. For all $n \geq 0$ and $\alpha=1,2,3,4$,

$$
c \phi_{2}\left(5^{\alpha} n+\lambda_{\alpha}\right) \equiv 0\left(\bmod 5^{\alpha}\right),
$$

where $\lambda_{\alpha}$ is the least positive reciprocal of 12 modulo $5^{\alpha}$.

In order to prove this theorem, we implement a finitization technique developed recently (cf. [3]). In essence, we prove that, for fixed $\alpha$,

$$
c \phi_{2}\left(5^{\alpha} n+\lambda_{\alpha}\right) \equiv 0\left(\bmod 5^{\alpha}\right) \quad \forall n
$$

if and only if

$$
c \phi_{2}\left(5^{\alpha} n+\lambda_{\alpha}\right) \equiv 0\left(\bmod 5^{\alpha}\right) \quad \forall n \leq C(\alpha),
$$

where $C(\alpha)$ is an explicit constant dependent on $\alpha$. We then compute all values of $c \phi_{2}$ needed to utilize the equivalence above. The development of $C(\alpha)$ requires the theory of modular forms as outlined below.

2. Determination of $C(\alpha)$. In this section, we use the theory of modular forms to determine the constant $C(\alpha)$. We do so by constructing a modular form whose Fourier coefficients inherit the congruence properties modulo $5^{\alpha}$ of $c \phi_{2}$ in the desired arithmetic progression. Then, thanks to a theorem of Sturm [10], we will be able to provide explicitly a constant $C(\alpha)$ such that if a congruence for the Fourier coefficients of our modular form (or equivalently, for $c \phi_{2}$ ) holds for all $n \leq C(\alpha)$, the congruence must hold for all $n$.

For a general introduction to the theory of modular forms, see [7]. For an exposition focused on the results we use below, see [3].

We now state Sturm's theorem [10].

THEOREM 2.1 (Sturm). If $f(z)=\sum_{n=0}^{\infty} a(n) q^{n}$ and $g(z)=\sum_{n=0}^{\infty} b(n) q^{n}$ are holomorphic modular forms of weight $k$ with respect to some congruence subgroup $\Gamma$ of $\mathrm{SL}_{2}(\mathbb{Z})$ with integer coefficients, then $f(z) \equiv g(z)(\bmod l)$ where $l$ is prime if and only if

$$
\operatorname{Ord}_{l}(f(z)-g(z))>\frac{k}{12}\left[\mathrm{SL}_{2}(\mathbb{Z}): \Gamma\right]
$$

where $\operatorname{Ord}_{l}(F(q)):=\min \{n \mid A(n) \not \equiv 0(\bmod l)\}$.

Sturm's theorem also holds when the prime $l$ is replaced by $5^{\alpha}$, or in fact by any positive integer. Thus, when we let $g(z)=0$, Sturm's theorem allows us to determine when the coefficients $a(n)$ of a holomorphic modular form have the property that $a(n) \equiv 0\left(\bmod 5^{\alpha}\right)$ for all $n$.

We are now ready to state the main result needed to prove Theorem 1.2. 
THEOREM 2.2. Suppose that $\alpha$ is a positive integer, and let

$$
C(\alpha):=6\left(b-1+4 \varepsilon \cdot 5^{\alpha-1}\right) 5^{\alpha-1}-\left\lceil\frac{b}{12}\right\rceil,
$$

where $b=b(\alpha)$ is the smallest integer greater than $4 \cdot 5^{\alpha-2}$ with $b \equiv 5^{\alpha}(\bmod 12)$, $\varepsilon=\varepsilon(\alpha)=1$ if $\alpha$ is odd, and $\varepsilon=\varepsilon(\alpha)=2$ if $\alpha$ is even. Then

$$
c \phi_{2}\left(5^{\alpha} n+\lambda_{\alpha}\right) \equiv 0\left(\bmod 5^{\alpha}\right) \quad \forall n
$$

if and only if

$$
c \phi_{2}\left(5^{\alpha} n+\lambda_{\alpha}\right) \equiv 0\left(\bmod 5^{\alpha}\right) \quad \forall n \leq C(\alpha),
$$

where $\lambda_{\alpha}$ is the least positive reciprocal of 12 modulo $5^{\alpha}$.

Proof. Let

$$
f(z)=\frac{\eta^{5}(2 z)}{\eta^{4}(z) \eta^{2}(4 z)} \eta^{b}\left(2 \cdot 5^{\alpha} z\right)\left(\frac{\eta^{5}(z)}{\eta(5 z)}\right)^{\varepsilon \cdot 5^{\alpha-1}}=\sum_{n=0}^{\infty} a(n) q^{n},
$$

where $\eta(z)$ is the Dedekind eta-function, defined by $\eta(z)=q^{1 / 24}(q ; q)_{\infty}, q=e^{2 \pi i z}$, $b=b(\alpha)$ is the smallest integer greater than $4 \cdot 5^{\alpha-2}$ with $b \equiv 5^{\alpha}(\bmod 12), \varepsilon=\varepsilon(\alpha)=1$ if $\alpha$ is odd, and $\varepsilon=\varepsilon(\alpha)=2$ if $\alpha$ is even.

Using results from [4, Theorems 3 and 5] on the properties of $\eta$-products, we find that $f(z)$ is a holomorphic modular form of weight $(b-1) / 2+2 \varepsilon \cdot 5^{\alpha-1}$ and character $\chi_{0}$, the trivial character, with respect to $\Gamma_{0}\left(16 \cdot 5^{\alpha}\right)$.

Notice that

$$
\left(\frac{\eta^{5}(z)}{\eta(5 z)}\right)^{\varepsilon \cdot 5^{\alpha-1}}=1+5^{\alpha} \sum_{n=1}^{\infty} h(n) q^{n},
$$

where the $h(n)$ are integers, and thus the Fourier coefficients of $f(z)$ are congruent to the Fourier coefficients of

$$
\frac{\eta^{5}(2 z)}{\eta^{4}(z) \eta^{2}(4 z)} \eta^{b}\left(2 \cdot 5^{\alpha} z\right)\left(\bmod 5^{\alpha}\right)
$$

Next, note that in terms of eta-functions,

$$
\sum_{n \geq 0} c \phi_{2}(n) q^{n}=q^{1 / 12} \frac{\eta^{5}(2 z)}{\eta^{4}(z) \eta^{2}(4 z)} .
$$

Thus, if we let

$$
q^{-2 b \cdot 5^{\alpha} / 24} \eta^{b}\left(2 \cdot 5^{\alpha} z\right)=\sum_{n=0}^{\infty} d\left(2 \cdot 5^{\alpha} n\right) q^{2 \cdot 5^{\alpha} n}
$$

then

$$
a\left(5^{\alpha} n+\lambda_{\alpha}+\frac{2 b \cdot 5^{\alpha}-2}{24}\right) \equiv \sum_{m=0}^{\infty} d\left(2 \cdot 5^{\alpha} m\right) c \phi_{2}\left(5^{\alpha} n+\lambda_{\alpha}-2 \cdot 5^{\alpha} m\right)\left(\bmod 5^{\alpha}\right) .
$$


Since $d(0)=1$, this becomes

$$
\begin{aligned}
a\left(5^{\alpha} n+\lambda_{\alpha}+\frac{2 b \cdot 5^{\alpha}-2}{24}\right) \equiv & c \phi_{2}\left(5^{\alpha} n+\lambda_{\alpha}\right) \\
& +\sum_{m=1}^{\infty} d\left(2 \cdot 5^{\alpha} m\right) c \phi_{2}\left(5^{\alpha} n+\lambda_{\alpha}-2 \cdot 5^{\alpha} m\right)\left(\bmod 5^{\alpha}\right) .
\end{aligned}
$$

By induction, it is easy to see that $c \phi_{2}\left(5^{\alpha} n+\lambda_{\alpha}\right) \equiv 0\left(\bmod 5^{\alpha}\right)$ for all $n \leq C(\alpha)$ if and only if $a\left(5^{\alpha} n+\lambda_{\alpha}+\left(2 b \cdot 5^{\alpha}-2\right) / 24\right) \equiv 0\left(\bmod 5^{\alpha}\right)$ for all $n \leq C(\alpha)$. Hence, we also have that $c \phi_{2}\left(5^{\alpha} n+\lambda_{\alpha}\right) \equiv 0\left(\bmod 5^{\alpha}\right)$ for all $n$ if and only if $a\left(5^{\alpha} n+\lambda_{\alpha}+\left(2 b \cdot 5^{\alpha}-2\right) / 24\right) \equiv$ $0\left(\bmod 5^{\alpha}\right)$ for all $n$.

Now notice that $\lambda_{\alpha}+\left(2 b \cdot 5^{\alpha}-2\right) / 24 \equiv 0\left(\bmod 5^{\alpha}\right)$ by hypothesis, so consider

$$
f_{1}(z)=f(z) \mid T_{5^{\alpha}}=\sum_{n=0}^{\infty} a\left(5^{\alpha} n\right) q^{n},
$$

which is also a holomorphic modular form of weight $(b-1) / 2+2 \varepsilon \cdot 5^{\alpha-1}$ and character $\chi_{0}$ with respect to $\Gamma_{0}\left(16 \cdot 5^{\alpha}\right)$. (See [7, pages 153-175] for a full explanation of the action of the Hecke operators $T_{p}$.) We find by Sturm's theorem that $a\left(5^{\alpha} n\right) \equiv 0\left(\bmod 5^{\alpha}\right)$ for all $n$ if and only if

$$
a\left(5^{\alpha} n\right) \equiv 0\left(\bmod 5^{\alpha}\right) \quad \forall n \leq \frac{\left((b-1) / 2+2 \varepsilon \cdot 5^{\alpha-1}\right)\left(16 \cdot 5^{\alpha}\right)}{12} \prod_{p \mid 10}\left(1+\frac{1}{p}\right) .
$$

Therefore, $c \phi_{2}\left(5^{\alpha} n+\lambda_{\alpha}\right) \equiv 0\left(\bmod 5^{\alpha}\right)$ for all $n$ if and only if the congruence holds for all $n \leq C(\alpha)$.

For certain values of $\alpha$, it is not difficult to make modest improvements to Theorem 1.2. In the case $\alpha=4$, this modest improvement will bring $C(\alpha)$ more comfortably within the realm of computational feasibility.

THEOREM 2.3. Let

$$
C(4):=198745 \text {. }
$$

Then

$$
c \phi_{2}(625 n+573) \equiv 0(\bmod 625) \quad \forall n
$$

if and only if

$$
c \phi_{2}(625 n+573) \equiv 0(\bmod 625) \quad \forall n \leq C(4) .
$$

Proof. Let

$$
f(z)=\frac{\eta^{5}(2 z)}{\eta^{4}(z) \eta^{2}(4 z)} \eta^{44}(625 z) \eta^{7}(1250 z) \eta^{10}(2500 z)\left(\frac{\eta^{5}(z)}{\eta(5 z)}\right)^{250}=\sum_{n=0}^{\infty} a(n) q^{n},
$$

where $q=e^{2 \pi i z}$. We find that $f(z)$ is a holomorphic modular form of weight 530 and character $\chi_{0}$, the trivial character, with respect to $\Gamma_{0}(2500)$. 
Notice that

$$
\left(\frac{\eta^{5}(z)}{\eta(5 z)}\right)^{250}=1+625 \sum_{n=1}^{\infty} h(n) q^{n}
$$

where the $h(n)$ are integers, and thus the Fourier coefficients of $f(z)$ are congruent to the Fourier coefficients of

$$
\frac{\eta^{5}(2 z)}{\eta^{4}(z) \eta^{2}(4 z)} \eta^{44}(625 z) \eta^{7}(1250 z) \eta^{10}(2500 z)(\bmod 625)
$$

Recalling that

$$
\sum_{n \geq 0} c \phi_{2}(n) q^{n}=q^{1 / 12} \frac{\eta^{5}(2 z)}{\eta^{4}(z) \eta^{2}(4 z)}
$$

if we let

$$
q^{-61250 / 24} \eta^{44}(625 z) \eta^{7}(1250 z) \eta^{10}(2500 z)=\sum_{n=0}^{\infty} d(625 n) q^{625 n}
$$

then

$$
a\left(625 n+573+\frac{61250-2}{24}\right) \equiv \sum_{m=0}^{\infty} d(625 m) c \phi_{2}(625 n+573-625 m)(\bmod 625) .
$$

Since $d(0)=1$, this becomes

$$
\begin{aligned}
a(625 n+573+2552) \equiv & c \phi_{2}(625 n+573) \\
& +\sum_{m=1}^{\infty} d(625 m) c \phi_{2}(625 n+573-625 m)(\bmod 625) .
\end{aligned}
$$

By induction, it is easy to see that $c \phi_{2}(625 n+573) \equiv 0(\bmod 625)$ for all $n \leq C(4)$ if and only if $a(625 n+573+2552) \equiv 0(\bmod 625)$ for all $n \leq C(4)$. Hence, we also have that $c \phi_{2}(625 n+573) \equiv 0(\bmod 625)$ for all $n$ if and only if $a(625 n+573+2552) \equiv$ $0(\bmod 625)$ for all $n$.

Now notice that $573+2552 \equiv 0(\bmod 625)$, so consider

$$
f_{1}(z)=f(z) \mid T_{625}=\sum_{n=0}^{\infty} a(625 n) q^{n},
$$

which is also a holomorphic modular form of weight 530 and character $\chi_{0}$ with respect to $\Gamma_{0}(2500)$. We find by Sturm's theorem that $a(625 n) \equiv 0(\bmod 625)$ for all $n$ if and only if

$$
a(625 n) \equiv 0(\bmod 625) \quad \forall n \leq \frac{(530)(2500)}{12} \prod_{p \mid 10}\left(1+\frac{1}{p}\right) .
$$

Therefore, $c \phi_{2}(625 n+573) \equiv 0(\bmod 625)$ for all $n$ if and only if the congruence holds for all $n \leq C(4)$. 
3. Calculating the needed values of $c \phi_{2}$. From the above discussion, we can prove the congruences desired for all $n$ after calculating the first $M$ values of $c \phi_{2}$, for any $M>5^{\alpha} C(\alpha)+\lambda_{\alpha}$. We calculate the necessary terms using recurrences.

The recurrences needed for $c \phi_{2}(m)$ are easily developed. Recurrences are suitable for calculating the values of $c \phi_{2}(m)$ for small $m$. This, of course, is the historical approach to the calculation of partition function values. For example, this was the technique used by MacMahon to compute the first 200 values of $p(m)$ [5, Table IV]. This same table was used by Ramanujan [8] in conjecturing several of the congruences in (1.5).

We now prove a result from which the necessary recurrences follow.

\section{THEOREM 3.1.}

$$
\left[\sum_{n \geq 0} c \phi_{2}(n) q^{n}\right]\left[\sum_{n \in \mathbb{Z}}(-1)^{n} q^{n^{2}}\right]=\left[\sum_{n \geq 0} p(n) q^{2 n}\right]\left[\sum_{n \in \mathbb{Z}} q^{n^{2}}\right] .
$$

Proof. From Jacobi's triple product identity [1, Theorem 2.8], we see that

$$
\sum_{n \in \mathbb{Z}}(-1)^{n} q^{n^{2}}=\left(q^{2} ; q^{2}\right)_{\infty}\left(q ; q^{2}\right)_{\infty}^{2}, \quad \sum_{n \in \mathbb{Z}} q^{n^{2}}=\frac{\left(q^{2} ; q^{2}\right)_{\infty}^{5}}{(q ; q)_{\infty}^{2}\left(q^{4} ; q^{4}\right)_{\infty}^{2}}
$$

Also, since

$$
\sum_{n \geq 0} p(n) q^{n}=\frac{1}{(q ; q)_{\infty}}
$$

it is clear that

$$
\sum_{n \geq 0} p(n) q^{2 n}=\frac{1}{\left(q^{2} ; q^{2}\right)_{\infty}}
$$

Then

$$
\begin{aligned}
{\left[\sum_{n \geq 0} c \phi_{2}(n) q^{n}\right]\left[\sum_{n \in \mathbb{Z}}(-1)^{n} q^{n^{2}}\right] } & =\frac{\left(q^{2} ; q^{4}\right)_{\infty}}{\left(q ; q^{2}\right)_{\infty}^{4}\left(q^{4} ; q^{4}\right)_{\infty}} \cdot\left(q^{2} ; q^{2}\right)_{\infty}\left(q ; q^{2}\right)_{\infty}^{2} \\
& =\frac{\left(q^{2} ; q^{2}\right)_{\infty}^{2}}{\left(q ; q^{2}\right)_{\infty}^{2}\left(q^{4} ; q^{4}\right)_{\infty}^{2}} \\
& =\frac{1}{\left(q^{2} ; q^{2}\right)_{\infty}} \cdot \frac{\left(q^{2} ; q^{2}\right)_{\infty}^{5}}{(q ; q)_{\infty}^{2}\left(q^{4} ; q^{4}\right)_{\infty}^{2}} \\
& =\left[\sum_{n \geq 0} p(n) q^{2 n}\right]\left[\sum_{n \in \mathbb{Z}} q^{n^{2}}\right] .
\end{aligned}
$$


From this theorem, we have the following recurrences:

$$
\begin{gathered}
c \phi_{2}(2 k)=p(k)+2 \sum_{m \geq 1}(-1)^{m+1} c \phi_{2}\left(2 k-m^{2}\right)+2 \sum_{m \geq 1} p\left(k-2 m^{2}\right), \\
c \phi_{2}(2 k+1)=2 \sum_{m \geq 1}(-1)^{m+1} c \phi_{2}\left(2 k+1-m^{2}\right)+2 \sum_{m \geq 0} p(k-2 m(m+1)) .
\end{gathered}
$$

Since $p(n)$ satisfies $p(n)=p(n-1)+p(n-2)-p(n-5)-p(n-7)+\cdots$, where the values in question are the pentagonal numbers, the above recurrences can easily be implemented to calculate several values of $c \phi_{2}$.

Using these recurrences, we have calculated the necessary 124, 216, 198 values of $c \phi_{2}$ on a Linux PC with $768 \mathrm{MB}$ of RAM and a $600 \mathrm{Mhz}$ Pentium III processor. The calculations, all performed modulo 625, were completed in approximately 147 hours of computing time.

With these calculations complete and the congruences checked modulo 625, Theorem 1.2 has been proven.

4. Closing remarks. While it would be nice to prove additional cases of (1.4) using this technique, it is clear that $C(\alpha)$ grows too rapidly to make such an approach feasible. For example, the proof of the $\alpha=5$ case of (1.4) would require the calculation of $C(5)=11279958$ values of $c \phi_{2}$ in the arithmetic progression $5^{5} n+\lambda_{5}$. Hence, we would have to calculate the first $3.5 \times 10^{10}$ values of $c \phi_{2}$ (approximately).

Certainly, a proof of Conjecture 1.1 via modular forms or generating function manipulations is still desired. This was originally requested in [9], and we renew that request here, given the new computational information that is now known about this partition function and the fact that Theorem 1.2 is proven.

ACKNOWLEDGEMENTs. The authors gratefully acknowledge Dr David Gallagher and Mr Robert Schumacher of Cedarville University for their invaluable assistance in the computation of values of $c \phi_{2}$. The first author was partially supported by NSF VIGRE Grant \#9977116.

\section{REFERENCES}

[1] G. E. Andrews, The Theory of Partitions, Encyclopedia of Mathematics and Its Applications, vol. 2, Addison-Wesley, Massachusetts, 1976.

[2] _ Generalized Frobenius partitions, Mem. Amer. Math. Soc. 49 (1984), no. 301, iv +44 .

[3] D. Eichhorn and K. Ono, Congruences for partition functions, Analytic Number Theory, vol. 1 (Allerton Park, IL, 1995), Progr. Math., vol. 138, Birkhäuser, Massachusetts, 1996, pp. 309-321.

[4] B. Gordon and K. Hughes, Multiplicative properties of $\eta$-products. II, A Tribute to Emil Grosswald: Number Theory and Related Analysis, Contemp. Math., vol. 143, American Mathematical Society, Rhode Island, 1993, pp. 415-430.

[5] G. H. Hardy and S. Ramanujan, Asymptotic formulae in combinatory analysis, Proc. London Math. Soc. 17 (1918), no. 2, 75-115.

[6] M. D. Hirschhorn and D. C. Hunt, A simple proof of the Ramanujan conjecture for powers of 5, J. Reine Angew. Math. 326 (1981), 1-17.

[7] N. Koblitz, Introduction to Elliptic Curves and Modular Forms, 2nd ed., Graduate Texts in Mathematics, vol. 97, Springer-Verlag, New York, 1993. 
[8] S. Ramanujan, Some properties of $p(n)$, the number of partitions of $n$, Proc. Cambridge Philos. Soc. 19 (1919), 207-210.

[9] J. Sellers, Congruences involving F-partition functions, Int. J. Math. Math. Sci. 17 (1994), no. 1, 187-188.

[10] J. Sturm, On the congruence of modular forms, Number Theory (New York, 1984-1985), Lecture Notes in Math., vol. 1240, Springer, Berlin, 1987, pp. 275-280.

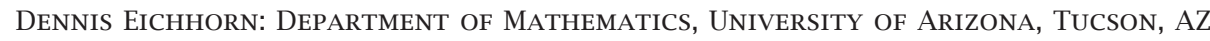
85721, USA

E-mail address: eichhorn@math. arizona.edu

James A. Sellers: Department of Mathematics, Penn State University, University PARK, PA 16802, USA

E-mail address: se11ersj@cedarvi11e.edu 


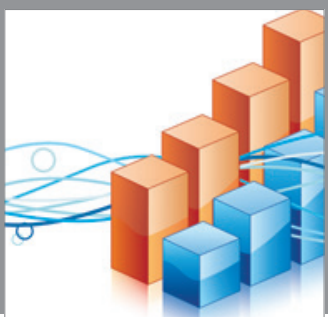

Advances in

Operations Research

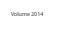

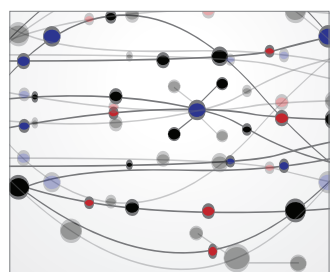

\section{The Scientific} World Journal
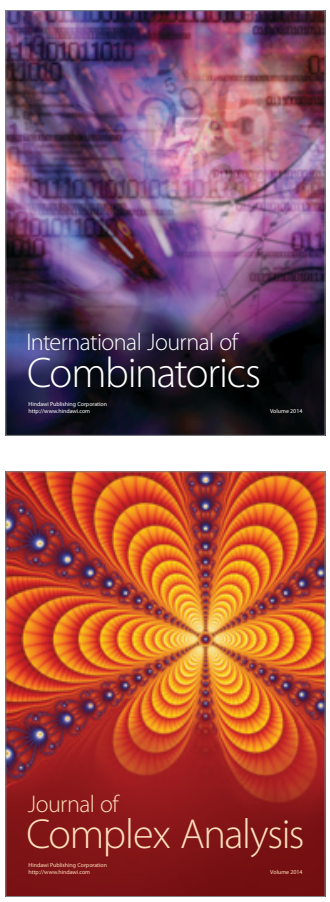

International Journal of

Mathematics and

Mathematical

Sciences
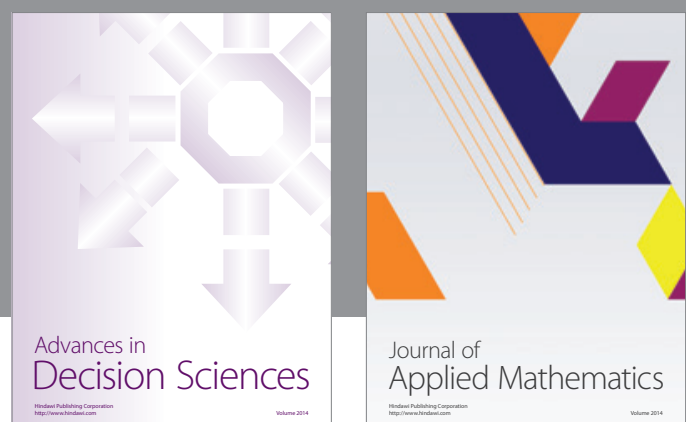

Journal of

Applied Mathematics
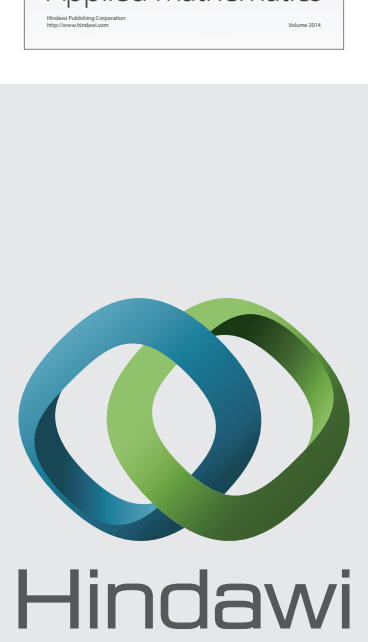

Submit your manuscripts at http://www.hindawi.com
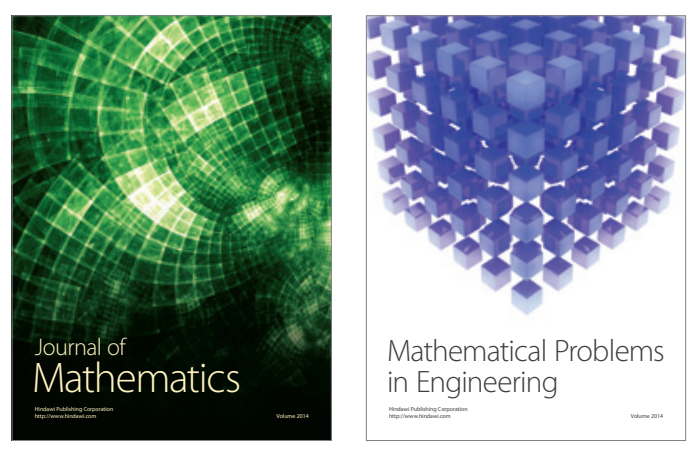

Mathematical Problems in Engineering
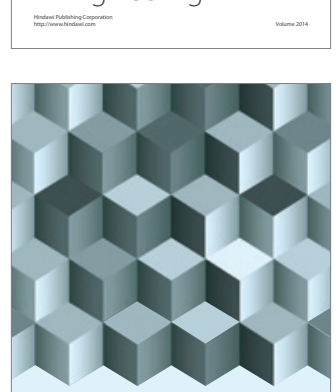

Journal of

Function Spaces
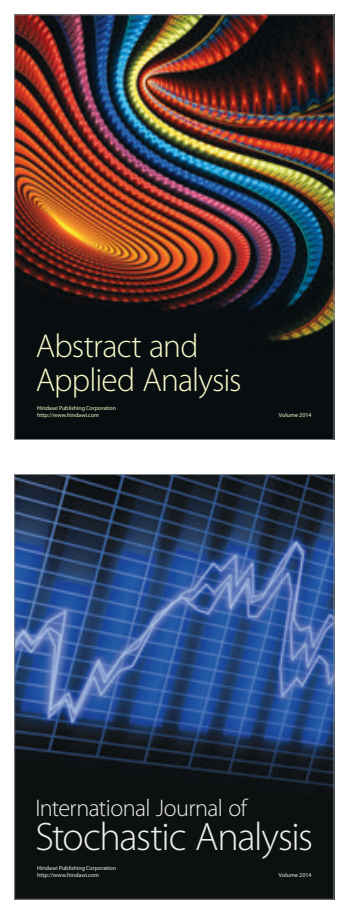

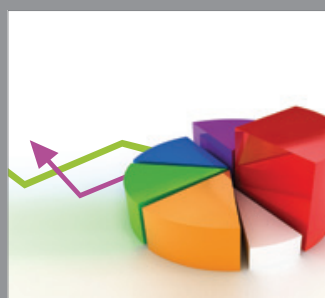

ournal of

Probability and Statistics

Promensencen
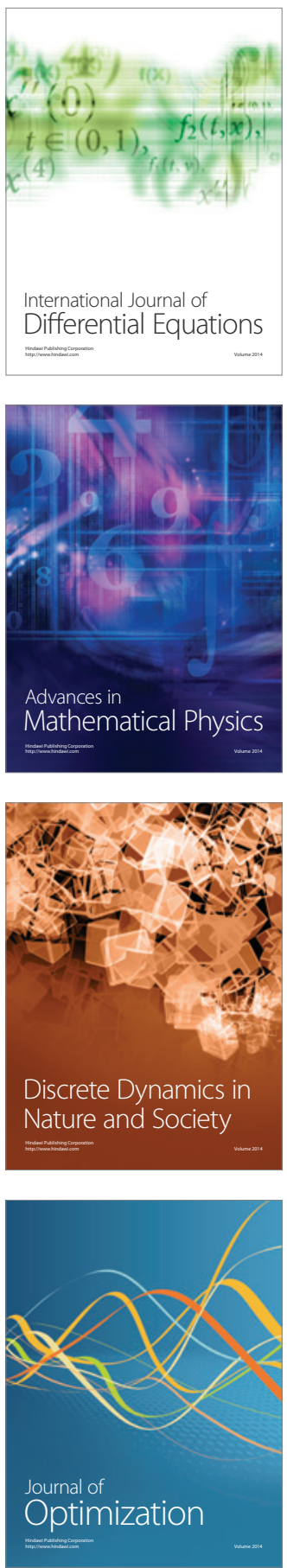